\title{
Riesz Basis Property of the Generalized Eigenvector System of A Timoshenko Beam ${ }^{1}$
}

\author{
Gen-Qi XU \\ Department of Mathematics of ShanXi University, TaiYuan 030006, P.R. China \\ Email:gqxu@sxu.edu.cn \\ De-Xing FENG \\ Academy of Mathematics and Systems Science, Chinese Academy of Sciences \\ Beijing 100080, P. R. China, Email:dxfeng@iss03.iss.ac.cn \\ Siu Pang YUNG \\ Department of Mathematics, University of Hong Kong, Hong Kong, P. R. China \\ Email: spyung@hkucc.hku.hk
}

\begin{abstract}
The Riesz basis property of the generalized eigenvector system of a Timoshenko beam with boundary feedback controls applied to two ends is studied in the present paper. The spectral property of the operator $\mathcal{A}$ determined by the closed loop system is investigated. It is shown that operator $\mathcal{A}$ has compact resolvent and generates a $C_{0}$ semigroup, and its spectrum consists of two branches and has two asymptotes under some conditions. Furthermore it is proved that the sequence of all generalized eigenvectors of the system principal operator forms a Riesz basis for the state Hilbert space.
\end{abstract}

Keywords. Timoshenko beam, boundary feedback, generalized eigenvector system, Riesz basis.

\section{Introduction}

It is well known that many flexible structure systems, such as robotic system, can be described by Timoshenko beam equation with appropriate boundary conditions (see Timoshenko,1954; Morgül, 1991). In the past decades, there exists an extensive mathematical and engineering literature on the Timoshenko beam model, and varied controllers for the system have been designed to realize the stabilization of the system. Among them, the so-called boundary controller is more easy to realize. Therefore the boundary control problem of Timoshenko

\footnotetext{
${ }^{1}$ This research was supported by the $\operatorname{NSFC}(60174008)$ and the Shanxi Natural Science Foundation
} 
beam system attracts much more attentions, and obtained a lot of important results, e.g., see Kim \& Renardy, 1987; Morgül,1991,1992; Feng et al.,1995, 1998; Shi et al, 1998; Geist \& McLaughlin, 2001; Pazoto\& Menzala, 2000. It is proved that if only one control force or moment is applied to one end, then the system can be asymptotically but not uniformly stabilized. If both control force and moment are applied to the one end or two ends of the system respectively, then the controlled system can be uniformly stabilized. However, these results do not give any information about the decay rate and expression of solution of the controlled system. In practice, we often need to make some quantitative analysis for the controlled system. If a system has the Riesz basis property, i.e., the sequence of all generalized eigenvectors of the principal operator for the system forms a Riesz basis of the state Hilbert space, then any solution of the system can be expressed in terms of the Riesz basis, and the system satisfies the spectrum determined growth assumption, and hence the stability is determined by the spectrum of the system principal operator.

In the past several years, on the generation problem of Riesz basis of the generalized eigenvector system of flexible structure with boundary control, many authors have made a great effort, e.g. see Balakrishnan, 1998; Shubov, 1999, 2002; Xu \& Feng, 2002; and the references therein. In Shubov 1999, the author proved the completeness of the generalized eigenvector sequence of the Timoshenko beam with some boundary feedback control. In the present paper, we give a counterexample to show that when the feedback gain is equal to the wave speed in this case, the sequence of the corresponding generalized eigenvectors is not complete.

In this paper we will consider the following homogeneous Timoshenko beam with boundary control:

$$
\left\{\begin{array}{l}
\rho \ddot{w}(x, t)-K\left(w^{\prime \prime}(x, t)-\varphi^{\prime}(x, t)\right)=0,0<x<\ell, \\
I_{\rho} \ddot{\varphi}(x, t)-E I \varphi^{\prime \prime}(x, t)-K\left(w^{\prime}(x, t)-\varphi(x, t)\right)=0,0<x<\ell, \\
w(0, t)=0, \quad \varphi(0, t)=\theta(t), \\
w^{\prime}(\ell, t)-\varphi(\ell, t)=u(t), \quad \varphi^{\prime}(\ell, t)=0
\end{array}\right.
$$

where $\theta(t)$ and $u(t)$ are control functions. The aim is to design feedback controllers such that the energy of the closed loop system is asymptotically stable. Here we adopt the following feedback control law:

$$
\dot{\theta}(t)=r\left(\varphi^{\prime}(0, t)-\theta(t)\right) \quad \text { and } \quad u(t)=-\beta \dot{w}(\ell, t), \quad r>0, \quad \beta>0 .
$$

To prove the stability of the closed loop system, the idea is to show that the sequence of the generalized eigenvectors of the closed loop system forms a Riesz basis in the energy space. For this purpose, we use Bari's Theorem (see, Young,1980). Here the key step in the use of Bari' Theorem is to find a reference 
basis of the state Hilbert space. We first introduce an auxiliary operator and prove that the sequence of the generalized eigenvectors of this auxiliary operator forms a Riesz basis. Then in terms of Bari's Theorem, we prove that the sequence of the generalized eigenvectors of the closed loop Timoshenko system forms a Riesz basis in Hilbert state space. Although the system we consider is with constant coefficients, but it is not difficult to extend to variable coefficient Timoshenko case.

The remaining part of the paper is arranged as follows. In the next section, we give a basic spectral analysis of $\mathcal{A}$ determined by the closed loop system (1.1) and (1.2), and describe the distribution of eigenvalues of $\mathcal{A}$. In section 3 , we introduce an auxiliary operator $\mathcal{A}_{0}$ which, in fact, is the principal part of $\mathcal{A}$, and prove that $\mathcal{A}_{0}$ has the Riesz basis property. Finally, in section 4 , by the asymptotic analysis of the operator $\mathcal{A}$, using Bari's Theorem, we prove $\mathcal{A}$ has the Riesz basis property, i.e., the generalized eigenvectors of the operator $\mathcal{A}$ forms a Riesz basis for the state Hilbert space.

\section{Spectral analysis of system operator}

In this section we set up the Hilbert state space, and write the closed loop system (1.1) and (1.2) into an evolutionary equation in the state Hilbert space. Further, we discuss the spectral property of the operator determined by the closed loop system.

Let us consider the following Timoshenko beam with boundary feedback control:

$$
\left\{\begin{array}{l}
\rho \ddot{w}(x, t)-K\left(w^{\prime \prime}(x, t)-\varphi^{\prime}(x, t)\right)=0,0<x<\ell \\
I_{\rho} \ddot{\varphi}(x, t)-E I \varphi^{\prime \prime}(x, t)-K\left(w^{\prime}(x, t)-\varphi(x, t)\right)=0,0<x<\ell \\
w(0, t)=0, \quad \varphi(0, t)=\theta(t), \quad \varphi^{\prime}(\ell, t)=0 \\
w^{\prime}(\ell, t)-\varphi(\ell, t)=-\beta \dot{w}(\ell, t), \quad \beta>0 \\
\dot{\theta}(t)=r\left(\varphi^{\prime}(0, t)-\theta(t)\right), \quad r>0
\end{array}\right.
$$

Define the state space

$$
\mathcal{H}=V_{0}^{1} \times L_{\rho}^{2}(0, \ell) \times H^{1}(0, \ell) \times L_{I_{\rho}}^{2}(0, \ell),
$$

where $V_{0}^{k}=\left\{\varphi \in H^{k}(0, \ell) \mid \varphi(0)=0\right\}, k=1,2$, and $H^{k}(0, \ell)$ is the usual Sobolev space of order $k$. For $Y_{1}=\left[w_{1}, z_{1}, \varphi_{1}, \psi_{1}\right]^{T}, Y_{2}=\left[w_{2}, z_{2}, \varphi_{2}, \psi_{2}\right]^{T} \in \mathcal{H}$, where and after the superscript $T$ denotes the transpose of matrix or vector, the inner product in $\mathcal{H}$ is defined by

$$
\begin{aligned}
\left\langle Y_{1}, Y_{2}\right\rangle= & \int_{0}^{\ell} K w_{1}^{\prime} \overline{w_{2}^{\prime}} d x+\int_{0}^{\ell} \rho z_{1} \overline{z_{2}} d x+\int_{0}^{\ell} E I \varphi_{1}^{\prime} \overline{\varphi_{2}^{\prime}} d x \\
& +\int_{0}^{\ell} I_{\rho} \psi_{1} \overline{\psi_{2}} d x+E I \varphi_{1}(0) \overline{\varphi_{2}(0)} .
\end{aligned}
$$


Define operator $\mathcal{A}$ in $\mathcal{H}$ :

$$
\begin{gathered}
\mathcal{A}\left(\begin{array}{c}
w \\
z \\
\varphi \\
\psi
\end{array}\right)=\left(\begin{array}{c}
z \\
\frac{K}{\rho}\left(w^{\prime \prime}-\varphi^{\prime}\right) \\
\psi \\
\frac{E I}{I_{\rho}} \varphi^{\prime \prime}+\frac{K}{I_{\rho}}\left(w^{\prime}-\varphi\right)
\end{array}\right), \\
\mathcal{D}(\mathcal{A})=\left\{[w, z, \varphi, \psi]^{\top} \in \mathcal{H} \mid w \in V_{0}^{2}, z \in V_{0}^{1}, \varphi \in H^{2}(0, \ell), \psi \in H^{1}(0, \ell)\right. \\
\left.\psi(0)=r\left(\varphi^{\prime}(0)-\varphi(0)\right), w^{\prime}(\ell)-\varphi(\ell)=-\beta z(\ell), \varphi^{\prime}(\ell)=0\right\} .
\end{gathered}
$$

Then the closed loop system (2.1) can be written as an evolutionary equation in $\mathcal{H}$ :

$$
\frac{d}{d t} Y(t)=\mathcal{A} Y(t), \quad \forall t>0,
$$

where $Y(t)=[w(\cdot, t), \dot{w}(\cdot, t), \varphi(\cdot, t), \dot{\varphi}(\cdot, t)]^{T}$.

Theorem 2.1 Let $\mathcal{A}$ be defined as above. Then $\mathcal{A}$ has compact resolvent and generates a $C_{0}$ semigroup.

Proof The proof is dierct and is omitted.

Now we are in a position to investigate the eigenvalue problem of $\mathcal{A}$. For $\lambda \in \mathbb{C}$, we solve eigenvalue-equation

$$
(\lambda I-\mathcal{A}) Y=0, \quad Y=[w, z, \varphi, \psi]^{\top} .
$$

This implies that $z(x)=\lambda w(x), \psi(x)=\lambda \varphi(x)$ and the function pair $(w(x), \varphi(x))$ satisfies the equations

$$
\left\{\begin{array}{l}
\rho \lambda^{2} w(x)-K\left(w^{\prime \prime}(x)-\varphi^{\prime}(x)\right)=0,0<x<\ell \\
I_{\rho} \lambda^{2} \varphi(x)-E I \varphi^{\prime \prime}(x)-K\left(w^{\prime}(x)-\varphi(x)\right)=0,0<x<\ell
\end{array}\right.
$$

with the boundary conditions

$$
\left\{\begin{array}{l}
w(0)=0, \quad \lambda \varphi(0)=r\left(\varphi^{\prime}(0)-\varphi(0)\right) \\
w^{\prime}(\ell)-\varphi(\ell)=-\beta \lambda \varphi(\ell), \quad \varphi^{\prime}(\ell)=0 .
\end{array}\right.
$$

For the sake of simplicity, for a fixed $\lambda \in \mathbb{C}$, set

$$
\rho_{1}^{2}=\frac{\rho}{K}, \quad \rho_{2}^{2}=\frac{I_{\rho}}{E I}, \quad a=\rho_{1}^{2} \lambda^{2}, \quad b=\rho_{2}^{2} \lambda^{2}+\frac{K}{E I}, \quad c=-\frac{K}{E I} .
$$

Denote by $\mu_{1}$ and $\mu_{2}$ the two roots of the quadratic equation

$$
\mu^{2}-(a+b+c) \mu+a b=0,
$$

i.e.,

$$
\mu_{1}=\mu_{1}(\lambda)=\frac{(a+b+c)+\sqrt{(a+b+c)^{2}-4 a b}}{2},
$$




$$
\mu_{2}=\mu_{2}(\lambda)=\frac{(a+b+c)-\sqrt{(a+b+c)^{2}-4 a b}}{2} .
$$

Assume that $\mu_{1} \neq \mu_{2}$, and define functions $w_{j}(\lambda, x), \varphi_{j}(\lambda, x)$ for $j=2,3,4$ as follows:

$$
\left\{\begin{array}{l}
w_{2}(\lambda, x)=\frac{b}{\mu_{1}-\mu_{2}}\left(\frac{\sinh \sqrt{\mu_{1}} x}{\sqrt{\mu_{1}}}-\frac{\sinh \sqrt{\mu_{2}} x}{\sqrt{\mu_{2}}}\right), \\
w_{3}(\lambda, x)=\frac{1}{\mu_{1}-\mu_{2}}\left(\left(\mu_{1}-b\right) \frac{\sinh \sqrt{\mu_{1}} x}{\sqrt{\mu_{1}}}-\left(\mu_{2}-b\right) \frac{\sinh \sqrt{\mu_{2}} x}{\sqrt{\mu_{2}}}\right) \\
w_{4}(\lambda, x)=\frac{1}{\mu_{1}-\mu_{2}}\left(\cosh \sqrt{\mu_{1}} x-\cosh \sqrt{\mu_{2}} x\right), \\
\varphi_{2}(\lambda, x)=\frac{1}{\mu_{1}-\mu_{2}}\left(-\left(\mu_{2}-b\right) \cosh \sqrt{\mu_{1}} x+\left(\mu_{1}-b\right) \cosh \sqrt{\mu_{2}} x\right), \\
\varphi_{3}(\lambda, x)=\frac{\left.\cosh \sqrt{\mu_{2}} x\right),}{\mu_{1}-\mu_{2}}\left(\cosh \sqrt{\mu_{1}} x-\cosh \left(\mu_{2}-a\right) \frac{\sinh \sqrt{\mu_{2}} x}{\sqrt{\mu_{2}}}\right) . \\
\varphi_{4}(\lambda, x)=\frac{1}{\mu_{1}-\mu_{2}}\left(\left(\mu_{1}-a\right) \frac{\sinh \sqrt{\mu_{1}} x}{\sqrt{\mu_{1}}}-\left(\mu_{2}\right.\right.
\end{array}\right.
$$

Then the general solution of (2.4) can be written as

$$
\begin{gathered}
w(x)=C_{2} w_{2}(\lambda, x)+C_{3} w_{3}(\lambda, x)+C_{4} w_{4}(\lambda, x), \\
\varphi(x)=C_{2} \varphi_{2}(\lambda, x)+C_{3} \varphi_{3}(\lambda, x)+C_{4} \varphi_{4}(\lambda, x),
\end{gathered}
$$

where $C_{2}, C_{3}, C_{4}$ are any constants to be determined. In order for $(w(x), \varphi(x))$ meet the boundary conditions (2.5), the constants $C_{2}, C_{3}, C_{4}$ must satisfy

$$
\begin{aligned}
& C_{4}=\frac{\lambda+r}{r} C_{2}, \\
& C_{2} a_{11}(\lambda)+C_{3} a_{12}(\lambda)=0, \\
& C_{2} a_{21}(\lambda)+C_{3} a_{22}(\lambda)=0,
\end{aligned}
$$

where

$$
\begin{aligned}
a_{11}(\lambda)= & w_{2}^{\prime}(\lambda, \ell)-\varphi_{2}(\lambda, \ell)+\lambda \beta w_{2}(\lambda, \ell) \\
& +\frac{\lambda+r}{r}\left[w_{4}^{\prime}(\lambda, \ell)-\varphi_{4}(\lambda, \ell)+\lambda \beta w_{4}(\lambda, \ell)\right], \\
a_{12}(\lambda)= & w_{3}^{\prime}(\lambda, \ell)-\varphi_{3}(\lambda, \ell)+\lambda \beta w_{3}(\lambda, \ell), \\
a_{21}(\lambda)= & \varphi_{2}^{\prime}(\lambda, \ell)+\frac{\lambda+r}{r} \varphi_{4}^{\prime}(\lambda, \ell), \\
a_{22}(\lambda)= & \varphi_{3}^{\prime}(\lambda, \ell) .
\end{aligned}
$$

Set

$$
\Gamma(\lambda)=\operatorname{det}\left(\begin{array}{ll}
a_{11}(\lambda) & a_{12}(\lambda) \\
a_{21}(\lambda) & a_{22}(\lambda)
\end{array}\right),
$$

Theorem 2.2. Let $w_{j}(\lambda, x), \varphi_{j}(\lambda, x)$ for $j=2,3,4$ and $\Gamma(\lambda)$ be defined as before. Then 
1) the spectrum $\sigma(\mathcal{A})$ of $\mathcal{A}$ consists of all eigenvalues of $\mathcal{A}$, and $\lambda \in \sigma(\mathcal{A})$ if and only if $\Gamma(\lambda)=0$;

2) let $\lambda \in \sigma(\mathcal{A})$, then the corresponding eigenvector can be chosen as

$$
\Phi_{\lambda}=C_{2}\left(\begin{array}{l}
\frac{1}{\lambda}\left[w_{2}(\lambda, x)+\frac{\lambda+r}{r} w_{4}(\lambda, x)\right] \\
w_{2}(\lambda, x)+\frac{\lambda+r}{r} w_{4}(\lambda, x) \\
\frac{1}{\lambda}\left[\varphi_{2}(\lambda, x)+\frac{\lambda+r}{r} \varphi_{4}(\lambda, x)\right] \\
\varphi_{2}(\lambda, x)+\frac{\lambda+r}{r} \varphi_{4}(\lambda, x)
\end{array}\right)+C_{3}\left(\begin{array}{c}
\frac{1}{\lambda} w_{3}(\lambda, x) \\
w_{3}(\lambda, x) \\
\frac{1}{\lambda} \varphi_{3}(\lambda, x) \\
\varphi_{3}(\lambda, x)
\end{array}\right),
$$

where $\left(C_{2}, C_{3}\right)$ is some nonzero solution of following linear equations

$$
\left\{\begin{array}{l}
C_{2} a_{11}(\lambda)+C_{3} a_{12}(\lambda)=0 \\
C_{2} a_{21}(\lambda)+C_{3} a_{22}(\lambda)=0 .
\end{array}\right.
$$

Furthermore, for each $\lambda \in \sigma(\mathcal{A})$, the eigen-subspace of $\mathcal{A}$ associated with $\lambda$ is of dimension one.

3) If $\beta \neq \rho_{1}$ and $r \rho_{2} \neq 1$, then the spectrum $\sigma(\mathcal{A})$ of $\mathcal{A}$ has two asymptotes. More precisely, there is a positive integer $N$ such that for each $n$ with $|n| \geq N$, two eigenvalues $\lambda_{n}^{(1)}$ and $\lambda_{n}^{(2)}$ of $\mathcal{A}$ are both simple and has the following asymptotic expression:

$$
\lambda_{n}^{(1)}=\left\{\begin{array}{lll}
\frac{1}{2 \rho_{1} \ell} \ln \left|\frac{\beta-\rho_{1}}{\beta+\rho_{1}}\right|+\frac{n \pi i}{\rho_{1} \ell}+O\left(\frac{1}{n}\right), & \text { if } & \beta-\rho_{1}>0, \\
\frac{1}{2 \rho_{1} \ell} \ln \left|\frac{\beta-\rho_{1}}{\beta+\rho_{1}}\right|+\frac{(2 n+1) \pi i}{2 \rho_{1} \ell}+O\left(\frac{1}{n}\right), & \text { if } & \beta-\rho_{1}<0,
\end{array}\right.
$$

and

$$
\lambda_{n}^{(2)}=\left\{\begin{array}{lll}
\frac{1}{2 \rho_{2} \ell} \ln \left|\frac{r \rho_{2}-1}{r \rho_{2}+1}\right|+\frac{n \pi i}{\rho_{2} \ell}+O\left(\frac{1}{n}\right), & \text { if } & r \rho_{2}-1>0, \\
\frac{1}{2 \rho_{2} \ell} \ln \left|\frac{r \rho_{2}-1}{r \rho_{2}+1}\right|+\frac{(2 n+1) \pi i}{2 \rho_{2} \ell}+O\left(\frac{1}{n}\right), & \text { if } & r \rho_{2}-1<0 .
\end{array}\right.
$$

Proof The assertions 1) and 2) are immediate. Here we only prove the assertion $3)$.

Obviously, $\Gamma(\lambda)$ is an entire function in $\lambda \in \mathbb{C}$. By using (2.7) and (2.8), a lengthy calculation shows that

$$
\lim _{\operatorname{Re} \lambda \rightarrow \infty} \frac{4 \Gamma(\lambda)}{\lambda}=\left[\rho_{2}+\frac{1}{r}\right]\left[1+\frac{\beta}{\rho_{1}}\right]
$$

and

$$
\begin{gathered}
\lim _{\operatorname{Re} \lambda \rightarrow-\infty} \frac{4 \Gamma(\lambda)}{\lambda}=\left[\rho_{2}-\frac{1}{r}\right]\left[1-\frac{\beta}{\rho_{1}}\right], \quad \text { if } \quad \rho_{1} \neq \beta, \quad r \rho_{2} \neq 1, \\
\lim _{\operatorname{Re} \lambda \rightarrow-\infty} 4 \Gamma(\lambda)=-1, \quad \text { if } \quad \rho_{1}=\beta, \quad r \rho_{2}=1,
\end{gathered}
$$

Then there is a $M>0$ large enough such that all zeros of $\Gamma(\lambda)$ lie in the strip $P_{M}=\{\lambda \in \mathbb{C} \mid-M \leq \operatorname{Re} \lambda \leq 0\}$. Note that the functions $\sinh \sqrt{\mu_{j}} \ell$ and 
$\cosh \sqrt{\mu_{j}} \ell$ are bounded in $\lambda \in P_{M}$. Hence for $\lambda \in P_{M}$ with $|\lambda|$ large enough, we have

$$
\frac{\Gamma(\lambda)}{\lambda}=\left[\cosh \rho_{1} \lambda \ell+\frac{\beta}{\rho_{1}} \sinh \rho_{1} \lambda \ell\right]\left[\rho_{2} \sinh \rho_{2} \lambda \ell+\frac{1}{r} \cosh \rho_{2} \lambda \ell\right]+O\left(\lambda^{-1}\right)
$$

Set

$$
G(\lambda)=\left[\cosh \rho_{1} \lambda \ell+\frac{\beta}{\rho_{1}} \sinh \rho_{1} \lambda \ell\right]\left[\rho_{2} \sinh \rho_{2} \lambda \ell+\frac{1}{r} \cosh \rho_{2} \lambda \ell\right] .
$$

The zeros of $G(\lambda)$ are

$$
\xi_{n}=\left\{\begin{array}{lll}
\frac{1}{2 \rho_{1} \ell} \ln \left|\frac{\beta-\rho_{1}}{\beta+\rho_{1}}\right|+\frac{n \pi i}{\rho_{1} \ell}, & \text { if } & \beta-\rho_{1}>0, \\
\frac{1}{2 \rho_{1} \ell} \ln \left|\frac{\beta-\rho_{1}}{\beta+\rho_{1}}\right|+\frac{(2 n+1) \pi i}{2 \rho_{1} \ell}, & \text { if } & \beta-\rho_{1}<0,
\end{array}\right.
$$

and

$$
\eta_{n}=\left\{\begin{array}{lll}
\frac{1}{2 \rho_{2} \ell} \ln \left|\frac{r \rho_{2}-1}{r \rho_{2}+1}\right|+\frac{n \pi i}{\rho_{2} \ell}, & \text { if } & r \rho_{2}-1>0, \\
\frac{1}{2 \rho_{2} \ell} \ln \left|\frac{r \rho_{2}-1}{r \rho_{2}+1}\right|+\frac{(2 n+1) \pi i}{2 \rho_{2} \ell}, & \text { if } & r \rho_{2}-1<0 .
\end{array}\right.
$$

Applying Rouche Theorem to (2.18), we can arrive that there is an integer $N$ such that for each $n$ with $|n|>N$, there exists only one zero $\lambda_{n}^{(1)}$ of $\Gamma(\lambda)$ in a small neighborhood of $\xi_{n}$, and similarly, there exists only one zero $\lambda_{n}^{(2)}$ of $\Gamma(\lambda)$ in a small neighborhood of $\eta_{n}$. Furthermore we have estimates

$$
\lambda_{n}^{(1)}=\xi_{n}+O\left(\xi_{n}^{-1}\right) \quad \text { and } \quad \lambda_{n}^{(2)}=\eta_{n}+O\left(\eta_{n}^{-1}\right) .
$$

By the first assertion we know that $\lambda_{n}^{(1)}, \lambda_{n}^{(2)} \in \sigma(\mathcal{A})$. Note that all the zeros of $G(\lambda)$ are simple. So for $n$ large enough, $\lambda_{n}^{(1)}, \lambda_{n}^{(2)} \in \sigma(\mathcal{A})$ are simple eigenvalues of $\mathcal{A}$. The proof is then complete.

Remark 2.3 If $1 \neq r \rho_{2}$ and $\beta \neq \rho_{1}$, then the spectrum of $\mathcal{A}$ distributes in a strip parallel to the imaginary axis, and has two asymptotes in general. But if $\left|\frac{r \rho_{2}-1}{r \rho_{2}+1}\right|=\left|\frac{\rho_{1}-\beta}{\rho_{1}+\beta}\right|$, then these two asymptotes degenerate the same one. In this case we cannot say that each eigenvalue of $\mathcal{A}$ with large modulus is simple or not.

When $1=r \rho_{2}$ and $\beta=\rho_{1}$, the function $G(\lambda)$ has no zero. It follows from (2.17) that $\Gamma(\lambda)$ has only finitely many zeros. Therefore in this case the system of the generalized eigenfunctions of $\mathcal{A}$ is not complete in the state space $\mathcal{H}$. This gives a counterexample of completeness result in Shubov (Shubov, 1999, 2002).

Theorem 2.4 There is no eigenvalue of $\mathcal{A}$ on the imaginary axis.

Proof Firstly a direct computation shows that $0 \in \rho(\mathcal{A})$.

We now suppose that the assertion is not true Then there is at least one $\lambda=i \tau \neq 0$ with $\tau \in R$ such that $\lambda \in \sigma(\mathcal{A})$. Let $Y=(w, \lambda w, \varphi, \lambda \varphi)$ is a corresponding eigenvector of $\mathcal{A}$. We have

$$
\operatorname{Re}\langle\mathcal{A} Y, Y\rangle=0=-K \beta|\lambda w(\ell)|^{2}-E I r\left|\varphi^{\prime}(0)-\varphi(0)\right|^{2},
$$


which implies that $w(\ell)=0$ and $\varphi(0)=\varphi^{\prime}(0)$. Since that $\lambda$ is an eigenvalue of $\mathcal{A}$, we have $\varphi^{\prime}(0)=\frac{\lambda+r}{r} \varphi(0)$, which holds only if $\varphi^{\prime}(0)=\varphi(0)=0$ because of $\lambda \neq 0$. So we have

$$
w(x)=C w_{3}(\lambda, x), \quad \varphi(x)=C \varphi_{3}(\lambda, x),
$$

where $C$ is a constant. It is easily seen that $w_{3}(\lambda, \ell) \neq 0$ as $\varphi_{3}^{\prime}(\lambda, \ell)=0$. So we obtain $C=0$, and hence $w=\varphi=0$, i.e., $Y=0$. This is a contradiction. Therefore the proof is complete.

\section{$3 \quad$ Auxiliary operator and its spectral property}

In order to investigate the Riesz basis property of the generalized eigenvector system of $\mathcal{A}$, we define an auxiliary operator $\mathcal{A}_{0}$ in $\mathcal{H}$ by

$$
\begin{aligned}
& \mathcal{A}_{0}\left(\begin{array}{c}
w \\
z \\
\varphi \\
\psi
\end{array}\right)=\left(\begin{array}{c}
z \\
\frac{K}{\rho} w^{\prime \prime} \\
\psi \\
\frac{E I}{I_{\rho}} \varphi^{\prime \prime}
\end{array}\right), \\
& \mathcal{D}\left(\mathcal{A}_{0}\right)=\left\{[w, z, \varphi, \psi]^{\top} \in \mathcal{H} \mid w \in V_{0}^{2}, z \in V_{0}^{1}, \varphi \in H^{2}(0, \ell),\right. \\
& \left.\psi \in H^{1}(0, \ell), \psi(0)=r \varphi^{\prime}(0), \varphi^{\prime}(\ell)=0, w^{\prime}(\ell)=-\beta z(\ell),\right\} .
\end{aligned}
$$

In the rest of this section, we study the property of $\mathcal{A}_{0}$. In the sequel, we denote by $Z$ the set of all integers, and always assume that $\rho_{1} \neq \rho_{2}$.

Theorem 3.1. Let $\mathcal{A}_{0}$ be defined by (3.1) and (3.2). Assume that $1 \neq r \rho_{2}, \beta \neq$ $\rho_{1}$, and set

$$
\begin{aligned}
& \tau_{1}= \begin{cases}\frac{1}{2 \ell} \ln \left|\frac{\rho_{1}-\beta}{\rho_{1}+\beta}\right|, & \text { if } \beta-\rho_{1}>0, \\
\frac{1}{2 \ell} \ln \left|\frac{\rho_{1}-\beta}{\rho_{1}+\beta}\right|+\frac{i \pi}{2 \ell}, & \text { if } \beta-\rho_{1}<0,\end{cases} \\
& \tau_{2}= \begin{cases}\frac{1}{2 \ell} \ln \left|\frac{r \rho_{2}-1}{r \rho_{2}+1}\right|, & \text { if } \quad r \rho_{2}-1>0, \\
\frac{1}{2 \ell} \ln \left|\frac{r \rho_{2}-1}{r \rho_{2}+1}\right|+\frac{i \pi}{2 \ell}, & \text { if } \quad r \rho_{2}-1<0 .\end{cases}
\end{aligned}
$$

Then

1) the spectrum $\sigma\left(\mathcal{A}_{0}\right)$ of $\mathcal{A}_{0}$ consists of all eigenvalues of $\mathcal{A}_{0}$, and is decomposed into two branches:

$$
\sigma\left(\mathcal{A}_{0}\right)=\left\{\xi_{n} \mid n \in Z\right\} \bigcup\left\{\eta_{n} \mid n \in Z\right\} \bigcup\{0\},
$$

where

$$
\rho_{1} \xi_{n}=\tau_{1}+i \frac{n \pi}{\ell}, \quad \rho_{2} \eta_{n}=\tau_{2}+i \frac{n \pi}{\ell}, \quad \forall n \in Z ;
$$


2) an eigenvector of $\mathcal{A}_{0}$ associated with $\xi_{n}$ is

$$
\Psi_{n}=\left[\xi_{n}^{-1} \sinh \left(\rho_{1} \xi_{n} x\right), \sinh \left(\rho_{1} \xi_{n} x\right), 0,0\right]^{\top}, \quad n \in Z,
$$

and similarly, an eigenvector of $\mathcal{A}_{0}$ corresponding to $\eta_{n}$ is

$\Phi_{n}=\left[0,0, \eta_{n}^{-1}\left[\sinh \left(\rho_{2} \eta_{n} x\right)+r \rho_{2} \cosh \left(\rho_{2} \eta_{n} x\right)\right], \sinh \left(\rho_{2} \eta_{n} x\right)+r \rho_{2} \cosh \left(\rho_{2} \eta_{n} x\right)\right]^{\top}$.

An eigenvector of $\mathcal{A}_{0}$ corresponding to 0 is

$$
F_{0}=[0,0,1,0]^{\top}
$$

3) the adjoint operator $\mathcal{A}_{0}^{*}$ of $\mathcal{A}_{0}$ is given by

$$
\begin{gathered}
\mathcal{A}_{0}^{*}\left(\begin{array}{c}
w(x) \\
z(x) \\
\varphi(x) \\
\psi(x)
\end{array}\right)=\left(\begin{array}{c}
-z(x) \\
-\frac{K}{\rho} w^{\prime \prime}(x) \\
-(\psi(x)-\psi(0)) \\
-\frac{E I}{I_{\rho}} \varphi^{\prime \prime}(x)
\end{array}\right) \\
\mathcal{D}\left(\mathcal{A}_{0}^{*}\right)=\left\{[w, z, \varphi, \psi]^{\top} \in \mathcal{H} \mid w \in V_{0}^{2}, z \in V_{0}^{1}, \varphi \in H^{2}, \psi \in H^{1}\right. \\
\left.w^{\prime}(\ell)=\beta z(\ell), \psi(0)=-r \varphi^{\prime}(0), \varphi^{\prime}(\ell)=0\right\} .
\end{gathered}
$$

4) the spectrum $\sigma\left(\mathcal{A}_{0}^{*}\right)$ of $\mathcal{A}_{0}^{*}$ is $\sigma\left(\mathcal{A}_{0}^{*}\right)=\overline{\sigma\left(\mathcal{A}_{0}\right)}=\sigma\left(\mathcal{A}_{0}\right)$. Moreover, if we set

$$
\begin{gathered}
\Psi_{n}^{*}=\left[\bar{\xi}_{n}^{-1} \sinh \left(\rho_{1} \bar{\xi}_{n} x\right),-\sinh \left(\rho_{1} \bar{\xi}_{n} x\right), 0,0\right]^{\top}, \quad n \in Z, \\
\Phi_{n}^{*}=\left[0,0, \bar{\eta}_{n}^{-1}\left[\sinh \left(\rho_{2} \bar{\eta}_{n} x\right)+r \rho_{2}\left(\cosh \left(\rho_{2} \bar{\eta}_{n} x\right)-1\right)\right],-\left[\sinh \left(\rho_{2} \bar{\eta}_{n} x\right)+r \rho_{2} \cosh \left(\rho_{2} \bar{\eta}_{n} x\right)\right]\right]^{\top}, \\
F_{0}^{*}=[0,0,1, r]^{\top},
\end{gathered}
$$

then $\left\{\Psi_{n}^{*}, \Phi_{n}^{*}, F_{0}^{*} \mid n \in Z\right\}$ is the eigenvector system of $\mathcal{A}_{0}^{*}$.

Proof We consider the eigenvalue problem of $\mathcal{A}_{0}$. Let $\lambda \in \mathbb{C}$ be an eigenvalue of $\mathcal{A}_{0}$, and $Y=[w, z, \varphi, \psi]^{\top} \in \mathcal{H}$ be an eigenvector of $\mathcal{A}_{0}$ corresponding to $\lambda$. Then $w(x)$ and $\varphi(x))$ satisfy

$$
\left\{\begin{array}{l}
\rho \lambda^{2} w(x)-K w^{\prime \prime}(x)=0,0<x<\ell, \\
I_{\rho} \lambda^{2} \varphi(x)-E I \varphi^{\prime \prime}(x)=0,0<x<\ell, \\
w(0)=0, \quad \lambda \varphi(0)=r \varphi^{\prime}(0) \\
w^{\prime}(\ell)=-\beta \lambda w(\ell), \quad \varphi^{\prime}(\ell)=0
\end{array}\right.
$$

Solving above equations, we obtain that

$$
w(x)=B \sinh \left(\rho_{1} \lambda x\right), \quad \varphi(x)=C\left[\sinh \left(\rho_{2} \lambda x\right)+r \rho_{2} \cosh \left(\rho_{2} \lambda x\right)\right],
$$


where $B$ and $C$ are constants. From the boundary condition of (3.10) it follows that

$$
B\left[\rho_{1} \lambda \cosh \left(\rho_{1} \lambda \ell\right)+\beta \lambda \sinh \left(\rho_{1} \lambda \ell\right)\right]=0,
$$

and

$$
C\left[\lambda \cosh \left(\rho_{2} \lambda \ell\right)+r \rho_{2} \lambda \sinh \left(\rho_{2} \lambda \ell\right)\right]=0 .
$$

If $C=0$ and $\beta \neq \rho_{1}$, then when $\lambda=0$, the equation (3.10) has a unique zero solution. As $\lambda \neq 0$, by solving equation (3.11) we get

$$
\lambda=\xi_{n}=\left\{\begin{array}{ll}
\frac{1}{2 \rho_{1} \ell} \ln \left|\frac{\beta-\rho_{1}}{\beta+\rho_{1}}\right|+i \frac{n \pi}{\rho_{1} \ell}, & \text { if } \quad \beta-\rho_{1}>0, \\
\frac{1}{2 \rho_{1} \ell} \ln \left|\frac{\beta-\rho_{1}}{\beta+\rho_{1}}\right|+i \frac{(2 n+1) \pi}{2 \rho_{1} \ell}, & \text { if } \quad \beta-\rho_{1}<0,
\end{array} \quad \forall n \in Z\right.
$$

If $B=0$ and $r \rho_{2} \neq 1$, then for $\lambda=0$, the equation (3.10) has a nonzero solution $\varphi(x)=1$. For $\lambda \neq 0$, from (3.12) we obtain

$$
\lambda=\eta_{n}=\left\{\begin{array}{ll}
\frac{1}{2 \rho_{2} \ell} \ln \left|\frac{r \rho_{2}-1}{r \rho_{2}+1}\right|+i \frac{n \pi}{\rho_{2} \ell}, & \text { if } r \rho_{2}-1>0, \\
\frac{1}{2 \rho_{2} \ell} \ln \left|\frac{r \rho_{2}-1}{r \rho_{2}+1}\right|+i \frac{(2 n+1) \pi}{2 \rho_{2} \ell}, & \text { if } r \rho_{2}-1<0,
\end{array} \quad \forall n \in Z .\right.
$$

So the point spectrum $\sigma_{p}\left(\mathcal{A}_{0}\right)$ of $\mathcal{A}_{0}$ is

$$
\sigma_{p}\left(\mathcal{A}_{0}\right)=\left\{\xi_{n} \mid n \in Z\right\} \bigcup\left\{\eta_{n} \mid n \in Z\right\} \bigcup\{0\} .
$$

It is easily to check that if $\lambda \notin \sigma_{p}\left(\mathcal{A}_{0}\right)$, then $\lambda \in \rho\left(\mathcal{A}_{0}\right)$, and so $\sigma\left(\mathcal{A}_{0}\right)=\sigma_{p}\left(\mathcal{A}_{0}\right)$.

Moreover, $\lambda=0$ is a simple eigenvalue of $\mathcal{A}_{0}$, and a corresponding eigenvector is $F_{0}=[0,0,1,0]$.

For $\xi_{n} \in \sigma\left(\mathcal{A}_{0}\right)$, a corresponding eigenvector is

$$
\Psi_{n}=\left[\xi_{n}^{-1} \sinh \left(\rho_{1} \xi_{n} x\right), \sinh \left(\rho_{1} \xi_{n} x\right), 0,0\right]^{\top},
$$

and for $\eta_{n} \in \mathcal{A}_{0}$, a corresponding eigenvector is $\Phi_{n}=\left[0,0, \eta_{n}^{-1}\left[\sinh \left(\rho_{2} \eta_{n} x\right)+r \rho_{2} \cosh \left(\rho_{2} \eta_{n} x\right)\right],\left(\sinh \left(\rho_{2} \eta_{n} x\right)+r \rho_{2} \cosh \left(\rho_{2} \eta_{n} x\right)\right)\right]^{\top}$.

The assertions 3) and 4) are direct. The proof is then complete.

Theorem 3.2. Let $\mathcal{H}_{1}$ and $H_{2}$ be two subspaces of $\mathcal{H}$ defined by

$$
\begin{aligned}
\mathcal{H}_{1} & =\left\{F=[w, z, 0,0]^{\top} \mid F \in \mathcal{H}\right\}, \\
\mathcal{H}_{2} & =\left\{F=[0,0, \varphi, \psi]^{\top} \mid F \in \mathcal{H}\right\} .
\end{aligned}
$$

Assume that $\tau_{1}, \tau_{2}, \xi_{n}$ and $\eta_{n}$ are defined as in Theorem 3.1. Then $\left\{\Psi_{n} \mid n \in Z\right\}$ forms a Riesz basis of $\mathcal{H}_{1}$, and $\left\{\Phi_{n} \mid n \in Z\right\} \bigcup\left\{F_{0}\right\}$ forms a Riesz basis of $\mathcal{H}_{2}$. Hence $\left\{\Psi_{n} \mid n \in Z\right\} \bigcup\left\{\Phi_{n} \mid n \in Z\right\} \bigcup\left\{F_{0}\right\}$ forms a Riesz basis of $\mathcal{H}$. 
Proof By virtue of Theorem 3.1, it is easily seen that for any $n, m \in Z$,

$$
\begin{gathered}
\left\langle F_{0}^{*}, \Psi_{n}\right\rangle=\left\langle F_{0}^{*}, \Phi_{n}\right\rangle=\left\langle\Phi_{n}^{*}, F_{0}\right\rangle=\left\langle\Psi_{n}^{*}, F_{0}\right\rangle=0, \\
=\left\langle\Phi_{n}^{*}, \Psi_{m}\right\rangle=\left\langle\Psi_{n}^{*}, \Phi_{m}\right\rangle=0, \quad \forall n \neq m, \\
\left\langle\Phi_{n}, \Phi_{n}^{*}\right\rangle=I_{\rho}\left(1+r^{2} \rho_{2}^{2}\right) \ell, \quad\left\langle\Psi_{n}, \Psi_{n}^{*}\right\rangle=\rho \ell, \quad\left\langle F_{0}^{*}, F_{0}\right\rangle=E I .
\end{gathered}
$$

Now we prove that $\left\{\Phi_{n} \mid n \in Z\right\} \bigcup\left\{F_{0}\right\}$ forms a Riesz basis of $\mathcal{H}_{2}$. To this end, for any $Y \in \mathcal{H}_{2}$, we define $a_{n}(Y)$ by

$$
\begin{aligned}
& I_{\rho}\left(1+r^{2} \rho_{2}^{2}\right) \ell a_{n}(Y)=\left\langle Y, \Phi_{n}^{*}\right\rangle \\
= & E I \int_{0}^{\ell} \rho_{2} \varphi^{\prime}(x) \overline{\left[\cosh \left(\rho_{2} \bar{\eta}_{n} x\right)+r \rho_{2} \sinh \left(\rho_{2} \bar{\eta}_{n} x\right)\right]} d x \\
& -I_{\rho} \int_{0}^{\ell} \psi(x) \overline{\left[\sinh \left(\rho_{2} \bar{\eta}_{n} x\right)+r \rho_{2} \cosh \left(\rho_{2} \bar{\eta}_{n} x\right)\right]} d x \\
= & \frac{\ell}{2} E I \rho_{2}\left[\mathcal{F}_{c, n}\left(\varphi^{\prime}\left[\cosh \left(\tau_{2} \cdot\right)+r \rho_{2} \sinh \left(\tau_{2} \cdot\right)\right]\right)+i \mathcal{F}_{s, n}\left(\varphi^{\prime}\left[\sinh \left(\tau_{2} \cdot\right)+r \rho_{2} \cosh (\tau \cdot)\right]\right)\right] \\
& \left.-\frac{\ell}{2} I_{\rho}\left[\mathcal{F}_{c, n}\left(\psi\left[\sinh \left(\tau_{2} \cdot\right)+r \rho_{2} \sinh \left(\tau_{2} \cdot\right)\right]\right)+i \mathcal{F}_{s, n}\left(\psi\left[\cosh \left(\tau_{2} \cdot\right)\right)+r \rho_{2} \sinh \left(\tau_{2} \cdot\right)\right]\right)\right],
\end{aligned}
$$

where $\mathcal{F}_{c, n}(g)$ and $\mathcal{F}_{s, n}(g)$ represent the Fourier coefficients of $g$ :

$$
\begin{aligned}
& \mathcal{F}_{c, n}(g)=\frac{2}{\ell} \int_{0}^{\ell} g(x) \cos \left(\frac{n \pi x}{\ell}\right) d x, \\
& \mathcal{F}_{s, n}(g)=\frac{2}{\ell} \int_{0}^{\ell} g(x) \sin \left(\frac{n \pi x}{\ell}\right) d x .
\end{aligned}
$$

Noticing that $\left\{\cos \left(\frac{n \pi x}{\ell}\right) \mid n \geq 0\right\}$ and $\left\{\sin \left(\frac{n \pi x}{\ell}\right) \mid n \geq 1\right\}$ are two normalized bases in $L^{2}[0, \ell]$, then we have

$$
\sum_{n=0}^{\infty}\left|\mathcal{F}_{c, n}(g)\right|^{2}<\infty, \quad \sum_{n=1}^{\infty}\left|\mathcal{F}_{s, n}(g)\right|^{2}<\infty
$$

Therefore

$$
\sum_{-\infty}^{\infty}\left|a_{n}(Y)\right|^{2}<\infty
$$

We define the subspace $\mathcal{H}_{0}$ of $\mathcal{H}_{2}$ by

$$
\mathcal{H}_{0}=\left\{Y \in \mathcal{H}_{2} \mid Y=\sum_{-\infty}^{\infty} a_{n}(Y) \Phi_{n}(x)+\left\langle Y, F_{0}^{*}\right\rangle F_{0}\right\} .
$$

Obviously, $\mathcal{H}_{0}$ is closed. If $\mathcal{H}_{0} \neq \mathcal{H}_{2}$, then there exists a $Y_{0} \in H_{2}, Y_{0}=$ $[0,0, \varphi(x), \psi(x)]^{\top} \neq 0$, such that $Y_{0} \perp \mathcal{H}_{0}$. So $Y_{0} \perp F_{0}, Y_{0} \perp \Phi_{n}, \forall n \in Z$. The equality $\left\langle Y_{0}, F_{0}\right\rangle=0$ implies that $\varphi(0)=0$. Then we define

$$
\mathcal{G}_{1}(\varphi)(x)= \begin{cases}\varphi(x), & \text { if } x \in[0, \ell], \\ \varphi(-x), & \text { if } x \in[-\ell, 0]\end{cases}
$$


and

$$
\mathcal{G}_{2}(\psi)(x)= \begin{cases}\psi(x), & \text { if } x \in[0, \ell], \\ -\psi(-x), & \text { if } x \in[-\ell, 0]\end{cases}
$$

We have

$$
\begin{aligned}
\left\langle Y_{0}, \Phi_{n}\right\rangle= & E I \int_{0}^{\ell} \varphi^{\prime} \rho_{2} \overline{\left[\cosh \left(\rho_{2} \eta_{n} x\right)+r \rho_{2} \sinh \left(\rho_{2} \eta_{n} x\right)\right]} d x \\
& +I_{\rho} \int_{0}^{\ell} \psi(x) \overline{\sinh \left(\rho_{2} \eta_{n} x\right) r \rho_{2} \cosh \left(\rho_{2} \eta_{n} x\right)} d x \\
= & \frac{E I \rho_{2}}{2} \int_{-\ell}^{\ell} \mathcal{G}_{1}\left(\varphi^{\prime}\right)(x) e^{\rho_{2} \bar{\eta}_{n} x} d x+\frac{E I r \rho_{2}^{2}}{2} \int_{-\ell}^{\ell} \mathcal{G}_{2}\left(\varphi^{\prime}\right)(x) e^{\rho_{2} \bar{\eta}_{n} x} d x \\
& +\frac{I_{\rho}}{2} \int_{-\ell}^{\ell} \mathcal{G}_{2}(\psi)(x) e^{\rho_{2} \bar{\eta}_{n} x}+\frac{I_{\rho} r \rho_{2}}{2} \int_{-\ell}^{\ell} \mathcal{G}_{1}(\psi)(x) e^{\rho_{2} \bar{\eta}_{n} x} d x=0 .
\end{aligned}
$$

This implies that

$$
\begin{aligned}
& \frac{E I \rho_{2}}{2} \varphi^{\prime}(x)+\frac{E I r \rho_{2}^{2}}{2} \varphi^{\prime}(x)+\frac{I_{\rho}}{2} \psi(x)+\frac{I_{\rho} r \rho_{2}}{2} \psi(x)=0, \quad \text { a.e., } x \in[0, \ell], \\
& \frac{E I \rho_{2}}{2} \varphi^{\prime}(x)-\frac{E I r \rho_{2}^{2}}{2} \varphi^{\prime}(x)-\frac{I_{\rho}}{2} \psi(x)+\frac{I_{\rho} r \rho_{2}}{2} \psi(x)=0, \quad \text { a.e., } x \in[0, \ell] .
\end{aligned}
$$

Solving the above algebraic equations, we obtain that $\varphi^{\prime}(x)=0$ and $\psi(x)=0$. Therefore $\varphi(x)=\psi(x)=0$, which contradicts to the hypothesis $Y_{0} \neq 0$. So we have $\mathcal{H}_{0}=\mathcal{H}_{2}$.

A similar argument can be used to prove that $\left\{\Psi_{n} \mid n \in Z\right\}$ forms a Riesz basis of $\mathcal{H}_{1}$. Noting that $\mathcal{H}=\mathcal{H}_{1}+\mathcal{H}_{2}$, we conclude that $\left\{\Psi_{n} \mid n \in Z\right\} \bigcup\left\{\Phi_{n} \mid\right.$ $n \in Z\} \bigcup\left\{F_{0}\right\}$ forms a Riesz basis of $\mathcal{H}$. The proof is then finished.

Corollary 3.3. If $\rho_{1}=\beta, r \rho_{2}=1$, then the spectrum $\sigma\left(\mathcal{A}_{0}\right)$ of $\mathcal{A}_{0}$ has only one element $\lambda=0$.

Proof Obviously, $\lambda=0$ is always an eigenvalue of $\mathcal{A}_{0}$ if $\beta=\rho_{1}, r \rho_{2}=1$. On the other hand, for any $\lambda \in \mathbb{C}, \lambda \neq 0,(3.11)$ and (3.12) with $B \neq 0$ or $C \neq 0$ can not hold. So $\sigma\left(\mathcal{A}_{0}\right)$ has a unique element $\lambda=0$.

\section{$4 \quad$ Riesz basis property of generalized eigenvector sys- tem of operator $\mathcal{A}$}

In this section we prove that the generalized eigenvector system of $\mathcal{A}$ forms a Riesz basis in $\mathcal{H}$. To begin with, we recall some notions and Bari's theorem. Let $\mathcal{H}$ be a separable Hilbert space, and $\left\{e_{n} \mid n \geq 1\right\}$ be a normalized basis of $\mathcal{H}$. A sequence of vectors, $\left\{f_{j} \mid j \geq 1\right\}$ in $\mathcal{H}$, is said to be a Riesz basis of $\mathcal{H}$ 
if there exists a bounded invertible linear operator $T$ in $\mathcal{H}$ such that $f_{n}=T e_{n}$ for all $n \geq 1$. Bari's theorem (e.g, see, Yuong, 1980) says that if an $\omega$-linear independent sequence $\left\{f_{n} \mid n \geq 1\right\}$ in $\mathcal{H}$ is quadratically near to a Riesz basis $\left\{g_{n} \mid n \geq 1\right\}$ in $\mathcal{H}$, i.e.,

$$
\sum_{n=0}^{\infty}\left\|g_{n}-f_{n}\right\|^{2}<\infty
$$

then $\left\{f_{n} \mid n \geq 1\right\}$ is a Riesz basis of $\mathcal{H}$. The following result can be found in Guo, 2001.

Proposition 4.1. Let $\mathcal{A}$ be a closed dense defined linear operator in $\mathcal{H}$. Assume that $\mathcal{A}$ is discrete, i.e., $\mathcal{A}$ has compact resolvent. Let $\left\{f_{n} \mid n \geq 1\right\}$ be a Riesz basis of $\mathcal{H}$. If there exists a sequence of generalized eigenvectors of $\mathcal{A},\left\{g_{n} \mid n \geq 1\right\}$ and a positive integer $N$ such that

$$
\sum_{n=N}^{\infty}\left\|f_{n}-g_{n}\right\|^{2}<\infty,
$$

then the system of all generalized eigenvectors of $\mathcal{A}$ forms a Riesz basis of $\mathcal{H}$.

Now we are in position to prove the following main result of this paper.

Theorem 4.2. Let $\mathcal{H}$ and $\mathcal{A}$ be defined as before, then the generalized eigenvector system forms a Riesz basis for $\mathcal{H}$.

Before going to the detail of the proof of theorem, we give the outline of the proof. Let $w_{j}(\lambda, x), \varphi_{j}(\lambda, x), j=2,3,4$ be defined by (2.8). Then for fixed $\lambda \in \mathbb{C}$, set

$$
Y_{j}(\lambda)=\left[\lambda^{-1} w_{j}(\lambda, x), w_{j}(\lambda, x), \lambda^{-1} \varphi_{j}(\lambda, x), \varphi(\lambda, x)\right]^{\top}, j=2,3,4 .
$$

Let $\lambda_{n}^{(1)}$ and $\lambda_{n}^{(2)}$ be the eigenvalues of $\mathcal{A}$ given by (2.14) and (2.15) respectively. According to (2.12) in theorem 2.2, the eigenvector associated with $\lambda_{n}^{(j)}$ is

$$
\Phi^{(j)}\left(\lambda_{n}^{(j)}\right)=C_{2}^{(j)}\left(\lambda_{n}^{(j)}\right)\left[Y_{2}\left(\lambda_{n}^{(j)}\right)+\frac{\lambda+r}{r} Y_{4}\left(\lambda_{n}^{(j)}\right)\right]+C_{3}^{(j)}\left(\lambda_{n}^{(j)}\right) Y_{3}\left(\lambda_{n}^{(j)}\right), \quad j=1,2,
$$

where $C_{2}^{(j)}, C_{3}^{(j)}$ satisfy (2.13) for $\lambda=\lambda_{n}^{(j)}$.

Let $\Phi_{n}=\Phi_{\xi_{n}}, \Psi_{n}=\Psi_{\eta_{n}}$ and $F_{0}$ be defined by (3.5), (3.6) and (3.7), respectively. We now choose $\left\{B_{n}^{1} \Phi_{n}, B_{n}^{(2)} \Psi_{n}, F_{0}, \mid n \in Z\right\}$ as a reference Riesz basis of $\mathcal{H}$, where $B_{n}^{(1)}$ and $B_{n}^{(2)}$ are some constants. We want to prove that with sutiable choice of $B_{n}^{(1)}$ and $B_{n}^{(2)}$, there exist a positive integer $N$ such that

$$
\sum_{|n|>N}^{\infty}\left\|\Phi^{(1)}\left(\lambda_{n}^{(1)}\right)-B_{n}^{(1)} \Psi_{\xi_{n}}\right\|^{2}<\infty
$$




$$
\sum_{|n|>N}^{\infty}\left\|\Phi^{(2)}\left(\lambda_{n}^{(2)}\right)-B_{n}^{(2)} \Phi_{\eta_{n}}\right\|^{2}<\infty .
$$

Proof of Theorem 4.2 Assume without loss of generality that $\rho_{1}>\rho_{2}$. Then for $\lambda \in \mathbb{C}$ with $|\lambda|$ large enough, we have

$$
\begin{aligned}
& \sqrt{\mu_{1}(\lambda)}=\rho_{1} \lambda+O\left(\lambda^{-1}\right), \\
& \sqrt{\mu_{2}(\lambda)}=\rho_{2} \lambda+O\left(\lambda^{-1}\right) .
\end{aligned}
$$

Particularly, for $\lambda=\lambda_{n}^{(1)}$, we have

$$
\begin{aligned}
& \sqrt{\mu_{1}\left(\lambda_{n}^{(1)}\right)}=\rho_{1} \lambda_{n}^{(1)}+O(1 / n)=\rho_{1} \xi_{n}+O(1 / n), \\
& \sqrt{\mu_{2}\left(\lambda_{n}^{(1)}\right)}=\rho_{2} \lambda_{n}^{(1)}+O(1 / n)=\rho_{2} \xi_{n}+O(1 / n),
\end{aligned}
$$

Similarly, for $\lambda=\lambda_{n}^{(2)}$, we have

$$
\begin{aligned}
& \sqrt{\mu_{1}\left(\lambda_{n}^{(2)}\right)}=\rho_{1} \lambda_{n}^{(2)}+O(1 / n)=\rho_{1} \eta_{n}+O(1 / n), \\
& \sqrt{\mu_{2}\left(\lambda_{n}^{(2)}\right)}=\rho_{2} \lambda_{n}^{(2)}+O(1 / n)=\rho_{2} \eta_{n}+O(1 / n) .
\end{aligned}
$$

Firstly, we choose the coefficient $C_{2}^{(j)}(\lambda)$ and $C_{3}^{(j)}(\lambda)$ in the expression of eigenvector (4.3) as follows:

$$
\begin{gathered}
C_{2}^{(1)}(\lambda)=\varphi_{3}^{\prime}(\lambda, \ell), \quad C_{3}^{(1)}(\lambda)=-\left[\varphi_{2}^{\prime}(\lambda, \ell)+\frac{\lambda+r}{r} \varphi_{4}^{\prime}(\lambda, \ell)\right] . \\
C_{2}^{(2)}(\lambda)=w_{3}^{\prime}(\lambda, \ell)-\varphi_{3}(\lambda, \ell)+\beta \lambda w_{3}(\lambda, \ell)
\end{gathered}
$$

and

$$
\begin{aligned}
C_{3}^{(2)}(\lambda)= & -\left[w_{2}^{\prime}(\lambda, \ell)-\varphi_{2}(\lambda, \ell)+\beta \lambda w_{2}(\lambda, \ell)\right. \\
& \left.+\frac{\lambda+r}{r}\left[w_{4}^{\prime}(\lambda, \ell)-\varphi_{4}(\lambda, \ell)+\beta \lambda w_{4}(\lambda, \ell)\right]\right] .
\end{aligned}
$$

Now for $\lambda \in \mathbb{C}$ with large modulus, we have the following estimates

$$
\begin{array}{lll}
\left|w_{2}^{\prime}(\lambda, x)\right|=O(1), & \left|w_{4}^{\prime}(\lambda, x)\right|=O(1 / \lambda), & \left|w_{3}^{\prime}(\lambda, x)\right|=O(1), \\
\left|\lambda w_{2}(\lambda, x)\right|=O(1), & \left|\lambda w_{4}(\lambda, x)\right|=O(1 / \lambda), & \left|\lambda w_{3}(\lambda, x)\right|=O(1), \\
\left|\varphi_{2}^{\prime}(\lambda, x)\right|=O(\lambda), & \left|\varphi_{4}^{\prime}(\lambda, x)\right|=O(1), & \left|\varphi_{3}^{\prime}(\lambda, x)\right|=O(1 / \lambda), \\
\left|\lambda \varphi_{2}^{\prime}(\lambda, x)\right|=O(\lambda), & \left|\lambda \varphi_{4}^{\prime}(\lambda, x)\right|=O(1), & \left|\lambda \varphi_{3}^{\prime}(\lambda, x)\right|=O(1 / \lambda) .
\end{array}
$$

This leads to that

$$
\left\|Y_{2}(\lambda)\right\|=O(1), \quad\left\|Y_{3}(\lambda)\right\|=O(1 / \lambda), \quad\left\|Y_{4}(\lambda)\right\|=O(1 / \lambda) .
$$

Since $\sqrt{\mu_{1}\left(\lambda_{n}^{(1)}\right)}=\rho_{1} \xi_{n}+O(1 / n)$, we have

$$
C_{2}^{(1)}\left(\lambda_{n}^{(1)}\right)=O\left(1 / \lambda_{n}^{(1)}\right), \quad C_{3}^{(1)}\left(\lambda_{n}^{(1)}\right)=O\left(\lambda_{n}^{(1)}\right)
$$


Similarly, we have $\sqrt{\mu_{2}\left(\lambda_{n}^{(2)}\right)}=\rho_{2} \eta_{n}+O(1 / n)$, and

$$
C_{2}^{(2)}\left(\lambda_{n}^{(2)}\right)=O(1), \quad C_{3}^{(2)}\left(\lambda_{n}^{(2)}\right)=O(1) .
$$

Therefore, $\Phi^{(1)}\left(\lambda_{n}^{(1)}\right)$ is expressed as

$$
\begin{aligned}
\Phi^{(1)}\left(\lambda_{n}^{(1)}\right)= & C_{2}^{(1)}\left(\lambda_{n}^{(1)}\right)\left[Y_{2}\left(\lambda_{n}^{(1)}\right)+\frac{\lambda_{n}^{(1)}+r}{r} Y_{4}\left(\lambda_{n}^{(1)}\right)\right]+C_{3}^{(1)}\left(\lambda_{n}^{(1)}\right) Y_{3}\left(\lambda_{n}^{(1)}\right) \\
= & \frac{C_{3}^{(1)}\left(\lambda_{n}^{(1)}\right)}{\lambda_{n}^{(1)}}\left(\begin{array}{c}
\frac{\mu_{1}\left(\lambda_{n}^{(1)}\right)-b\left(\lambda_{n}^{(1)}\right)}{\mu_{1}\left(\lambda_{n}^{(1)}\right)-\mu_{2}\left(\lambda_{n}^{(1)}\right)} \frac{1}{\sqrt{\mu_{1}\left(\lambda_{n}^{(1)}\right)}} \sinh \left(\sqrt{\mu_{1}\left(\lambda_{n}^{(1)}\right) x}\right) \\
\frac{\mu_{1}\left(\lambda_{n}^{(1)}\right)-b\left(\lambda_{n}^{(1)}\right)}{\mu_{1}\left(\lambda_{n}^{(1)}\right)-\mu_{2}\left(\lambda_{n}^{(1)}\right)} \frac{\lambda_{n}^{(1)}}{\sqrt{\mu_{1}\left(\lambda_{n}^{(1)}\right)}} \sinh \left(\sqrt{\mu_{1}\left(\lambda_{n}^{(1)}\right) x}\right) \\
0 \\
0
\end{array}\right)+G_{1}\left(\lambda_{n}^{(1)}\right) .
\end{aligned}
$$

where $C_{2}^{(1)}(\lambda)$ and $C_{3}^{(1)}(\lambda)$ are given by (4.4), and $G_{1}\left(\lambda_{n}^{(1)}\right)$ is given by

$$
\begin{gathered}
G_{1}(\lambda)=\frac{C_{3}^{(1)}(\lambda)}{\lambda}\left(\begin{array}{c}
-\frac{\mu_{2}(\lambda)-b(\lambda)}{\mu_{1}(\lambda)-\mu_{2}(\lambda)} \frac{1}{\sqrt{\mu_{2}(\lambda)}} \sinh \left(\sqrt{\mu_{2}(\lambda)} x\right) \\
-\frac{\mu_{2}(\lambda)-b(\lambda)}{\mu_{1}(\lambda)-\mu_{2}(\lambda)} \frac{\lambda}{\sqrt{\mu_{2}(\lambda)}} \sinh \left(\sqrt{\mu_{1}(\lambda)} x\right) \\
\varphi_{3}(\lambda, x) \\
\lambda \varphi_{3}(\lambda, x) \\
+C_{2}^{(1)}(\lambda)\left(Y_{2}(\lambda)+\frac{\lambda+r}{r} Y_{4}(\lambda)\right),
\end{array}\right)
\end{gathered}
$$

with the estimate $\left\|G_{1}\left(\lambda_{n}^{(1)}\right)\right\|=O\left(1 / \lambda_{n}^{(1)}\right)$.

Similarly, the eigenvector $\Phi^{(2)}\left(\lambda_{n}^{(2)}\right)$ is expressed as

$$
\begin{aligned}
\Phi^{(2)}\left(\lambda_{n}^{(2)}\right)= & C_{2}^{(2)}\left(\lambda_{n}^{(2)}\right)\left[Y_{2}\left(\lambda_{n}^{(2)}\right)+\frac{\lambda_{n}^{(2)}+r}{r} Y_{4}\left(\lambda_{n}^{(2)}\right)\right]+C_{3}^{(2)}\left(\lambda_{n}^{(2)}\right) Y_{3}\left(\lambda_{n}^{(2)}\right) \\
0 & \left.C_{2}^{(2)}\left(\lambda_{n}^{(2)}\right)\left(\begin{array}{c}
0 \\
0 \\
\frac{\mu_{1}-b}{\lambda\left(\mu_{1}-\mu_{2}\right)} \cosh \left(\sqrt{\mu_{2}} x\right)+\frac{\mu_{1}-b}{\mu_{1}-\mu_{2}} \frac{1}{r \sqrt{\mu_{2}}} \sinh \left(\sqrt{\mu_{2}} x\right) \\
\frac{\mu_{1}-b}{\mu_{1}-\mu_{2}} \cosh \left(\sqrt{\mu_{2}} x\right)+\frac{\mu_{1}-b}{\mu_{1}-\mu_{2}} \frac{\lambda}{r \sqrt{\mu_{2}}} \sinh \left(\sqrt{\mu_{2}} x\right)
\end{array}\right)\right|_{\lambda=\lambda_{n}^{(2)}} \\
& +G_{2}\left(\lambda_{n}^{(2)}\right) .
\end{aligned}
$$

where $C_{2}^{(2)}(\lambda)$ and $C_{3}^{(2)}(\lambda)$ are given by (4.5) and (4.6) respectively, and $G_{2}(\lambda)$ is given by

$$
G_{2}(\lambda)=C_{3}^{(2)}(\lambda) Y_{3}(\lambda)+C_{2}^{(2)}(\lambda) Y_{4}(\lambda)+C_{2}^{(2)}(\lambda)\left(\begin{array}{c}
\frac{1}{\lambda}\left[w_{2}(\lambda, x)+\frac{\lambda}{r} w_{4}(\lambda, x)\right] \\
w_{2}(x)+\frac{\lambda}{r} w_{4}(\lambda, x) \\
p_{3}(\lambda) \\
p_{4}(\lambda)
\end{array}\right)
$$


where

$$
\begin{aligned}
p_{3}(\lambda)= & -\frac{\mu_{2}(\lambda)-b(\lambda)}{\lambda\left(\mu_{1}(\lambda)-\mu_{2}(\lambda)\right)} \cosh \left(\sqrt{\mu_{1}(\lambda)} x\right)+\frac{\mu_{1}(\lambda)-a(\lambda)}{\mu_{1}(\lambda)-\mu_{2}(\lambda)} \frac{1}{r \sqrt{\mu_{1}(\lambda)}} \sinh \left(\sqrt{\mu_{1}(\lambda)} x\right) \\
& -\frac{c}{\mu_{1}(\lambda)-\mu_{2}(\lambda)} \frac{1}{r \sqrt{\mu_{1}(\lambda)}} \sinh \left(\sqrt{\mu_{1}(\lambda)} x\right), \\
p_{4}(\lambda)= & -\frac{\mu_{2}(\lambda)-b(\lambda)}{\mu_{1}(\lambda)-\mu_{2}(\lambda)} \cosh \left(\sqrt{\mu_{1}(\lambda)} x\right)+\frac{\mu_{1}(\lambda)-a(\lambda)}{\mu_{1}(\lambda)-\mu_{2}(\lambda)} \frac{\lambda}{r \sqrt{\mu_{1}(\lambda)}} \sinh \left(\sqrt{\mu_{1}(\lambda)} x\right) \\
& -\frac{c}{\mu_{1}(\lambda)-\mu_{2}(\lambda)} \frac{\lambda}{r \sqrt{\mu_{1}(\lambda)}} \sinh \left(\sqrt{\mu_{1}(\lambda)} x\right) .
\end{aligned}
$$

Moreover, we have the estimate $\left\|G_{2}\left(\lambda_{n}^{(2)}\right)\right\|=O\left(1 / \lambda_{n}^{(2)}\right)$.

Set

$$
\widehat{\Psi}(\lambda)=\frac{C_{3}^{(1)}(\lambda)}{\lambda}\left(\begin{array}{c}
\frac{\mu_{1}(\lambda)-b(\lambda)}{\mu_{1}(\lambda)-\mu_{2}(\lambda)} \frac{1}{\sqrt{\mu_{1}(\lambda)}} \sinh \left(\sqrt{\mu_{1}(\lambda)} x\right) \\
\frac{\mu_{1}(\lambda)-b(\lambda)}{\mu_{1}(\lambda)-\mu_{2}(\lambda)} \frac{\lambda}{\sqrt{\mu_{1}(\lambda)}} \sinh \left(\sqrt{\mu_{1}(\lambda)} x\right) \\
0 \\
0
\end{array}\right),
$$

and

$$
\widehat{\Phi}(\lambda)=\left.C_{2}^{(2)}(\lambda)\left(\begin{array}{c}
0 \\
0 \\
\frac{\mu_{1}-b}{\lambda\left(\mu_{1}-\mu_{2}\right)} \cosh \left(\sqrt{\mu_{2}} x\right)+\frac{\mu_{1}-b}{\mu_{1}-\mu_{2}} \frac{1}{r \sqrt{\mu_{2}}} \sinh \left(\sqrt{\mu_{2}} x\right) \\
\frac{\mu_{1}-b}{\mu_{1}-\mu_{2}} \cosh \left(\sqrt{\mu_{2}} x\right)+\frac{\mu_{1}-b}{\mu_{1}-\mu_{2}} \frac{\lambda_{n}^{(2)}}{r \sqrt{\mu_{2}}} \sinh \left(\sqrt{\mu_{2}} x\right)
\end{array}\right)\right|_{\mu_{j}=\mu_{j}(\lambda)} .
$$

Then we have

$$
\Phi^{(1)}\left(\lambda_{n}^{(1)}\right)=\widehat{\Psi}\left(\lambda_{n}^{(1)}\right)+G_{1}\left(\lambda_{n}^{(1)}\right), \quad \Phi^{(2)}\left(\lambda_{n}^{(2)}\right)=\widehat{\Phi}\left(\lambda_{n}^{(2)}\right)+G_{2}\left(\lambda_{n}^{(2)}\right) .
$$

In order to esitmate the vectors $\widehat{\Psi}\left(\lambda^{(1)}\right)$ and $\widehat{\Phi}\left(\lambda_{n}^{(2)}\right)$, we denote

$$
A(\lambda)=\frac{\mu_{1}(\lambda)-b(\lambda)}{\mu_{1}(\lambda)-\mu_{2}(\lambda)}
$$

Thus

$$
\widehat{\Psi}\left(\lambda_{n}^{(1)}\right)=A\left(\lambda_{n}^{(1)}\right) \frac{C_{3}^{(1)}\left(\lambda_{n}^{(1)}\right)}{\lambda_{n}^{(1)}}\left(\begin{array}{c}
\frac{1}{\sqrt{\mu_{1}\left(\lambda_{n}^{(1)}\right)}} \sinh \left(\sqrt{\mu_{1}\left(\lambda_{n}^{(1)}\right)} x\right) \\
\frac{\lambda_{n}^{(1)}}{\sqrt{\mu_{1}\left(\lambda_{n}^{(1)}\right)}} \sinh \left(\sqrt{\mu_{1}\left(\lambda_{n}^{(1)}\right)} x\right) \\
0 \\
0
\end{array}\right)
$$

and

$$
\widehat{\Phi}\left(\lambda_{n}^{(2)}\right)=A\left(\lambda_{n}^{(2)}\right) C_{2}^{(2)}\left(\lambda_{n}^{(2)}\right)\left(\begin{array}{c}
0 \\
0 \\
q_{3}\left(\lambda_{n}^{(2)}\right) \\
q_{4}\left(\lambda_{n}^{(2)}\right)
\end{array}\right)
$$


where

$$
\begin{aligned}
& q_{3}(\lambda)=\frac{1}{\lambda} \cosh \left(\sqrt{\mu_{2}(\lambda)} x\right)+\frac{1}{r \sqrt{\mu_{2}(\lambda)}} \sinh \left(\sqrt{\mu_{2}(\lambda)} x\right), \\
& q_{4}(\lambda)=\cosh \left(\sqrt{\mu_{2}(\lambda)} x\right)+\frac{\lambda}{r \sqrt{\mu_{2}(\lambda)}} \sinh \left(\sqrt{\mu_{2}(\lambda)} x\right) .
\end{aligned}
$$

Set

$$
\begin{aligned}
& B_{n}^{(1)}:=A\left(\lambda_{n}^{(1)}\right) \frac{C_{3}^{(1)}\left(\lambda_{n}^{(1)}\right)}{\lambda_{n}^{(1)}}, \quad B_{n}^{(2)}:=A\left(\lambda_{n}^{(2)}\right) C_{2}^{(2)}\left(\lambda_{n}^{(2)}\right), \\
& \alpha_{n}^{(1)}:=\sqrt{\mu_{1}\left(\lambda_{n}^{(1)}\right)}-\rho_{1} \xi_{n}, \quad \alpha_{n}^{(2)}:=\sqrt{\mu_{2}\left(\lambda^{(2)}\right)}-\rho_{2} \eta_{n} .
\end{aligned}
$$

Obviously, $\alpha_{n}^{(j)}=O(1 / n)$ for $j=1,2$.

Now let $\Psi_{n}$ and $\Phi_{n}$ be defined as in Theorem 3.1. Then, when $|n| \geq N$, we have

$$
\begin{aligned}
& \left\|\widehat{\Psi}\left(\lambda_{n}^{(1)}\right)-B_{n}^{(1)} \Psi_{n}\right\|^{2} \\
& =\left|B_{n}^{(1)}\right|^{2}\left[\int_{0}^{\ell} K\left|\cosh \left(\sqrt{\mu_{1}} x\right)-\cosh \left(\rho_{1} \xi_{n} x\right)\right|^{2} d x\right. \\
& \left.+\int_{0}^{\ell} \rho\left|\frac{\lambda_{n}^{(1)}}{\sqrt{\mu_{1}}} \sinh \left(\sqrt{\mu_{1}} x\right)-\frac{1}{\rho_{1}} \sinh \left(\rho_{1} \xi_{n} x\right)\right|^{2} d x\right] \\
& =\left|B_{n}^{(1)}\right|^{2}\left[\int_{0}^{\ell} K \mid\left[\cosh \left(\alpha_{n}^{(1)} x\right)-1\right] \cosh \left(\rho_{1} \xi_{n} x\right)\right. \\
& +\left.\sinh \left(\alpha_{n}^{(1)} x\right) \sinh \left(\rho_{1} \xi_{n} x\right)\right|^{2} d x+\int_{0}^{\ell} \rho \mid \frac{\lambda_{n}^{(1)}}{\sqrt{\mu_{1}}} \sinh \left(\alpha_{n}^{(1)} x\right) \cosh \left(\rho_{1} \xi_{n} x\right) \\
& \left.-\left.\left(\frac{\lambda_{n}^{(1)}}{\sqrt{\mu_{1}}} \cosh \left(\alpha_{n}^{(1)} x\right)-\frac{1}{\rho_{1}}\right) \sinh \left(\rho_{1} \xi_{n} x\right)\right|^{2} d x\right] \\
& \leq\left|B_{n}^{(1)}\right|^{2}\left[K \int_{0}^{\ell}\left[\left|\cosh \left(\alpha_{n}^{(1)} x\right)-1\right|^{2}+\left|\sinh \left(\alpha_{n}^{(1)} x\right)\right|^{2}\right] \times\right. \\
& \times\left[\left|\cosh \left(\rho_{1} \xi_{n} x\right)\right|^{2}+\left|\sinh \left(\rho_{1} \xi_{n} x\right)\right|^{2}\right] d x \\
& +\rho \int_{0}^{\ell}\left(\left|\frac{\lambda_{n}^{(1)}}{\sqrt{\mu_{1}}} \cosh \left(\rho_{1} \xi_{n} x\right)\right|^{2}+\left|\sinh \left(\rho_{1} \xi_{n} x\right)\right|^{2}\right) \times \\
& \left.\times\left(\left|\sinh \left(\alpha_{n}^{(1)} x\right)\right|^{2}+\left|\frac{\lambda_{n}^{(1)}}{\sqrt{\mu_{1}}} \cosh \left(\alpha_{n}^{(1)} x\right)-\frac{1}{\rho_{1}}\right|^{2}\right) d x\right] \\
& \leq M_{1}\left|\alpha_{n}^{(1)}\right|^{2} e^{2\left|\alpha_{n}^{(1)}\right| \ell}
\end{aligned}
$$

where we have used the fact that $\left|B_{n}^{(1)}\right|^{2}$ is uniformly bounded, and

$$
\left\|\widehat{\Phi}\left(\lambda_{n}^{(2)}\right)-\frac{B_{n}^{(2)}}{r \rho_{2}} \Phi_{n}\right\|^{2}
$$




$$
\begin{aligned}
&=\left|B_{n}^{(2)}\right|^{2}\left[\int_{0}^{\ell} E I \mid \frac{\sqrt{\mu_{2}}}{\lambda_{n}^{(2)}} \sinh \left(\sqrt{\mu_{2}} x\right)+\frac{1}{r} \cosh \left(\sqrt{\mu_{2}} x\right)\right. \\
&-\left.\frac{1}{r}\left[\cosh \left(\rho_{2} \eta_{n} x\right)+r \rho_{2} \sinh \left(\rho_{2} \eta_{n} x\right)\right]\right|^{2} d x \\
&+\int_{0}^{\ell} I_{\rho} \mid \cosh \left(\sqrt{\mu_{2}} x\right)+\frac{\lambda}{r \sqrt{\mu_{2}}} \sinh \left(\sqrt{\mu_{2}} x\right) \\
&\left.-\left.\frac{1}{r \rho_{2}}\left[\sinh \left(\rho_{2} \eta_{n} x\right)+r \rho_{2} \cosh \left(\rho_{2} \eta_{n} x\right)\right]\right|^{2} d x+E I\left|\frac{1}{\lambda_{n}^{(2)}}-\frac{1}{r \rho_{2} \eta_{n}} r \rho_{2}\right|^{2}\right] \\
& \leq\left|B_{n}^{(2)}\right|^{2}\left(E I \int _ { 0 } ^ { \ell } \left[\left|\frac{\sqrt{\mu_{2}}}{\lambda_{n}^{(2)}} \cosh \left(\alpha_{n}^{(2)} x\right)-\rho_{2}\right|^{2}\right.\right. \\
&\left.+\left|\sinh \left(\alpha_{n}^{(2)} x\right)\right|^{2}+\left|\cosh \left(\alpha_{n}^{(2)} x\right)-1\right|^{2}+\left|\sinh \left(\alpha_{n}^{(2)} x\right)\right|^{2}\right] \times \\
& \times\left.|| \sinh \left(\rho_{2} \eta_{n} x\right)\right|^{2}+\left|\frac{\sqrt{\mu_{2}}}{\lambda_{n}^{(2)}} \cosh \left(\rho_{2} \eta_{n} x\right)\right|^{2}+\left|\frac{1}{r} \cosh \left(\rho_{2} \eta_{n} x\right)\right|^{2} \\
&\left.+\left|\frac{1}{r} \sinh \left(\rho_{2} \eta_{n} x\right)\right|^{2}\right] d x+I_{\rho} \int_{0}^{\ell}\left[\left|\cosh \left(\alpha_{n}^{(2)} x\right)-1\right|^{2}+\left|\sinh \left(\alpha_{n}^{(2)} x\right)\right|^{2}\right. \\
&\left.+\left|\frac{\lambda_{n}^{(2)}}{r \sqrt{\mu_{2}}} \cosh \left(\alpha_{n}^{(2)} x\right)-\frac{1}{r \rho_{2}}\right|^{2}+\left|\sinh \left(\alpha_{n}^{(2)} x\right)\right|^{2}\right] \times \\
& \times\left[\left|\cosh \left(\rho_{2} \eta_{n} x\right)\right|^{2}+\left|\sinh \left(\rho_{2} \eta_{n} x\right)\right|^{2}+\left|\sinh \left(\rho_{2} \eta_{n} x\right)\right|^{2}\right. \\
&\left.\left.+\left|\frac{\lambda_{n}^{(2)}}{r \sqrt{\mu_{2}}} \cosh \left(\rho_{2} \eta_{n} x\right)\right|^{2}\right] d x+E I\left|\frac{1}{\lambda_{n}^{(2)}}-\frac{1}{r \rho_{2} \eta_{n}} r \rho_{2}\right|^{2}\right) \\
& \leq M_{2}\left|\alpha_{n}^{(2)}\right|^{2} e^{2 \mid \alpha_{n}(2)} \mid \ell \\
&
\end{aligned}
$$

Note that

$$
\begin{aligned}
& \Phi\left(\lambda_{n}^{(1)}\right)-B_{n}^{(1)} \Psi_{n}=\left[\widehat{\Psi}\left(\lambda_{n}^{(1)}\right)-B_{n}^{(1)} \Psi_{n}\right]+G_{1}\left(\lambda_{n}^{(1)}\right), \\
& \Phi\left(\lambda_{n}^{(2)}\right)-B_{n}^{(2)} /\left(r \rho_{2}\right) \Phi_{n}=\left[\widehat{\Phi}\left(\lambda_{n}^{(2)}\right)-B_{n}^{(2)} /\left(r \rho_{2}\right) \Phi_{n}\right]+G_{2}\left(\lambda_{n}^{(2)}\right) .
\end{aligned}
$$

Hence there exists a positive integer $N$ such that when $|n| \geq N$, we have

$$
\begin{aligned}
& \left\|\Psi\left(\lambda_{n}^{(1)}\right)-B_{n}^{(1)} \Psi_{n}\right\|^{2} \leq 2\left[M_{1}\left|\alpha_{n}^{(1)}\right|^{2} e^{2\left|\alpha_{n}^{(1)}\right| \ell}+\left\|G_{1}\left(\lambda_{n}^{(1)}\right)\right\|^{2}\right] \leq \frac{\widehat{M}_{1}}{n^{2}}, \\
& \left\|\Psi\left(\lambda_{n}^{(2)}\right)-\frac{B_{n}^{(2)}}{r \rho_{2}} \Phi_{n}\right\|^{2} \leq 2\left[M_{1}\left|\alpha_{n}^{(2)}\right|^{2} e^{2\left|\alpha_{n}^{(2)}\right| \ell}+\left\|G_{1}\left(\lambda_{n}^{(2)}\right)\right\|^{2}\right] \leq \frac{\widehat{M}_{2}}{n^{2}},
\end{aligned}
$$

where $\widehat{M}_{1}$ and $\widehat{M}_{2}$ are independent on $n$. Therefore, we obtain

$$
\sum_{|n| \geq N}\left\|\Psi\left(\lambda_{n}^{(1)}\right)-B_{n}^{(1)} \Psi_{n}\right\|^{2}+\sum_{|n| \geq N}\left\|\Psi\left(\lambda_{n}^{(2)}\right)-\frac{B_{n}^{(2)}}{r \rho_{2}} \Phi_{n}\right\|^{2}<\infty .
$$

Since it holds that

$$
A\left(\lambda_{n}^{(j)}\right) \neq 0, \quad \text { and } \quad A\left(\lambda_{n}^{(j)}\right) \rightarrow 1, \quad(\text { as } n \rightarrow \infty), \quad \forall j=1,2,
$$




$$
0<\inf _{|n|>N, n \in Z}\left|B_{n}^{(j)}\right| \leq \sup _{|n|>N, n \in Z}\left|B_{n}^{(j)}\right|<\infty, \quad j=1,2,
$$

we can assume without loss of generality that, for $|n|<N$,

$$
B_{n}^{(1)}=1, \quad B_{n}^{(2)} /\left(r \rho_{2}\right)=1, \quad|n|<N,
$$

then $\left\{B_{n}^{(1)} \Psi_{n} \mid n \in Z\right\} \bigcup\left\{\frac{B_{n}^{(2)}}{r \rho_{2}} \Phi_{n} \mid n \in Z\right\} \bigcup\left\{F_{0}\right\}$ is also a Riesz basis for $\mathcal{H}$. By virtue of Proposition 4.1, the generalized eigenvector system of $\mathcal{A}$ forms a Riesz basis for $\mathcal{H}$. The proof is then complete.

Remark 4.3. We know from Theorem 2.3 and 4.2 that there is a positive integer $N$ such that $\lambda_{n}^{(1)}, \lambda_{n}^{(2)}$ for $|n| \geq N$ are simple eigenvalues of $\mathcal{A}, \Psi\left(\lambda_{n}^{(1)}\right)$ and $\Phi\left(\lambda_{n}^{(2)}\right)$ are eigenvectors of $\mathcal{A}$ associated with $\lambda_{n}^{(1)}$ and $\lambda_{n}^{(2)}$, respectively, and the sequence

$$
\left\{\Psi\left(\lambda_{n}^{(1)}\right)|n \in Z,| n \mid \geq N\right\} \bigcup\left\{\Phi\left(\lambda_{n}^{(2)}\right)|n \in Z,| n \mid \geq N\right\}
$$

is a basis sequence. So the number of the elements in set

$$
\sigma_{1}(\mathcal{A})=\sigma(\mathcal{A}) \backslash\left\{\lambda_{n}^{(1)}, \lambda_{n}^{(2)},|n \in Z,| n \mid \geq N\right\}
$$

is less than number $4 N-1$. Although we do not know exact number of the non-simple eigenvalues of $\mathcal{A}$ in the set $\sigma_{1}(\mathcal{A})$, we know that the exact dimension of subspace corresponding to $\sigma_{1}(\mathcal{A})$ is $4 N-1$.

Corollary 4.4 Let $\mathcal{H}$ and $\mathcal{A}$ be defined as before, and $T(t)$ be the $C_{0}$ semigroup generated by $\mathcal{A}$. Assume that $\sigma_{1}(\mathcal{A})=\left\{\mu_{1}, \mu_{2}, \cdots, \mu_{k}\right\}$. Then when $\beta-\rho_{1} \neq 0$, $r \rho_{2}-1 \neq 0$, the solution of the system determined by (2.1) with initial data $F \in \mathcal{H}$ can be expressed as

$$
\begin{aligned}
T(t) F= & \sum_{j=1}^{k} \sum_{i=1}^{s_{j}} e^{\mu_{j} t} P_{s_{j}, i}(F, t) \Phi_{j, i}+\sum_{|n| \geq N} e^{\lambda_{n}^{(1)} t}\left\langle F, \Psi^{*}\left(\lambda_{n}^{(1)}\right)\right\rangle \Psi\left(\lambda_{n}^{(1)}\right) \\
& +\sum_{|n| \geq N} e^{\lambda_{n}^{(2)} t}\left\langle F, \Phi^{*}\left(\lambda_{n}^{(2)}\right)\right\rangle \Phi\left(\lambda_{n}^{(2)}\right) .
\end{aligned}
$$

From Theorem 2.3 we know that the lines $\operatorname{Re} \lambda=\operatorname{Re} \frac{\tau_{1}}{\rho_{1}}$ and $\operatorname{Re} \lambda=\operatorname{Re} \frac{\tau_{2}}{\rho_{2}}$ are two asymptotes of the spectrum of $\mathcal{A}$. Denote

$$
\omega_{0}=\max \left\{\operatorname{Re} \frac{\tau_{1}}{\rho_{1}}, \operatorname{Re} \frac{\tau_{2}}{\rho_{2}}\right\},
$$

obviously, $\omega_{0}<0$. Since $\mathcal{A}$ is dissipative operator and $\sigma(\mathcal{A}) \cap i R=\emptyset$, we can obtain the following corollary. 
Corollary 4.5. Let $\mathcal{H}$ and $\mathcal{A}$ be defined as before. If $\beta \neq \rho_{1}$ and $r \rho_{2} \neq 1$, then the system determined by (2.1) satisfies the spectrum determined growth assumption in the state space $\mathcal{H}$, i.e.,

$$
\omega(T)=s(\mathcal{A})=\max _{\lambda \in \sigma(\mathcal{A})}\left\{\omega_{0}, \operatorname{Re} \lambda\right\}<0
$$

and hence the closed loop system is exponentially stable.

\section{References}

1 Balakrishnan, A. V.(1998) Dynamics and control of articulated anisotropic Timoshenko beams, Dynamics and control of distributed systems, 121-201, Cambridge Univ. Press, Cambridge.

2 Feng, D. X. and Zhang, W. T.(1995) Nonlinear feedback control of Timoshenko beam, Science in China, Ser. A, 38, 8, 918-927.

3 Feng, D. X., Shi, D. H. and Zhang, W. T.(1998) Boundary feedback stabilization of Timoshenko beam with boundary dissipation, Science in China, Ser. A, 415, 483-490.

4 Geist, B. and McLaughlin, J. R. (2001) Asymptotic formulas for the eigenvalues of the Timoshenko beam, J. Math. Anal. Appl., 253, 2, 341-380.

5 Kim, J. U. and Renardy,Y.(1987) Boundary control of the Timoshenko Beam, SIAM. J. Control and Optimization, 25, 6, 1417-1429.

6 Morgül,Ö.(1991) Boundary control of a Timoshenko beam attached to a rigid body: Planar motion, Int. J. Control, 54, (1991), 763-791.

7 Morgü, Ö. (1992) Dynamic boundary control of the Timoshenko beam, Automatica, 28, 6, 1255-1260.

8 Pazoto, A. F. and Menzala, G. P. (2000) Uniform stabilization of a nonlinear beam model with thermal effects and nonlinear boundary dissipation, Funkcial. Ekvac., 43), 2, 339-360.

9 Shi, D. H. and Feng, D. X.(1998) Feedback stabilization of a Timoshenko beam with an end mass, Int. J. Control, 69, 2, 285-300.

10 Shubov, M. A.(1999) Spectral operators generated by Timoshenko beam model, Systems Control Lett., 38, 4/5, 249-258.

11 Shubov, M. A. (2002) Asymptotic and spectral analysis of the spatially nonhomogeneous Timoshenko beam model, Math. Nachr., 241, 125-162.

12 Guo,B. Z.(2001) The Riesz basis property of Discrete operators and application to a Euler-Bernolli beam equation with boundary linear feedback control, IMA J. Math. Control \& Information,18, 241-251.

13 Timoshenko, S.(1954) Vibration Problems in Engineering, Van Norstrand, New York, 1955. 
$14 \mathrm{Xu}, \mathrm{G} . \mathrm{Q}$ and Feng, D. X.(2002) The Riesz basis property of a Timoshenko beam with boundary feedback and application, IMA J. Appl. Math., 67, 357-370.

15 Young, R. M.(1980) An Introduction to Nonharmonic Fourier Series, Academic Press, pp45 (Theorem 15). 